\title{
Propagation Characteristics of Laser-induced Acoustic Sources in Hybrid Anechoic Wind Tunnels
}

\author{
Máté Szőke ${ }^{\mathrm{a}, *}$, William Devenport ${ }^{\mathrm{a}}$ \\ ${ }^{a}$ Center for Renewable Energy and Aerodynamic Technology (CREATe), \\ Aerospace and Ocean Engineering, Virginia Tech, Blacksburg, Virginia, USA, 24061
}

\begin{abstract}
The propagation characteristics of an acoustic point source generated using laser-induced plasma (LIP) were investigated experimentally. Experiments were performed in a Kevlar-walled hybrid anechoic wind tunnel (HAWT) where the sound of the LIP was measured using a 251-element microphone array, while the flow speed in the empty test section was varied. The time instant of the LIP formation was also captured. The far field sound pressure was assessed through arrival times (source to microphones) and pressure correction levels, and these quantities were compared against a commonly used shear layer refraction model. A detailed uncertainty assessment is presented on the arrival times and pressure levels. It was found that the time domain analysis was limited by the sampling rate of the analog-to-digital converter regardless of the flow speed. The uncertainty of the pressure levels was limited by the uncertainty of the microphones at low flow speeds, while they increased with flow speed at shallow observer angles. The high-speed Schlieren imaging of the LIP was performed, which revealed that the sound of the LIP reaches the far field microphones over a shorter time duration than modeled because the wave speed was initially supersonic. The discrepancy was found to be comparable to the temporal resolution of the aeroacoustic experiments. The discrepancy between the experimental and theoretical arrival times was found to increase with flow speed, and they were nearly independent of the azimuth angles. The discrepancy between the experimental and theoretical pressure correction ratio was found to be uniform for most observer locations. With an increase in flow speed, the discrepancy became positive at large, and negative at low polar angles. The sound refraction at the Kevlar wall did not change the frequency content of the sound over the investigated range of frequencies $(1-10 \mathrm{kHz})$.
\end{abstract}

Keywords: Laser-induced breakdown, shear-layer, refraction, aeroacoustic correction, uncertainty quantification 


\section{Introduction}

Acoustic measurements are usually performed in anechoic, low-noise environments, where the noise source of interest can be well isolated. In aeroacoustic experiments, however, the presence of a flow is necessary to generate the noise source of interest. Unfortunately, the flow itself also introduces a series of complications to such experiments. Conventionally, aeroacoustic experiments are performed using open-jet wind tunnels whose test volume is located inside an anechoic chamber 1. While this approach is favorable from an acoustic experimentation point of view because it separates microphones from the flow, its aerodynamic performances are often limited, for example, the jet is deflected when studying lifting bodies, such as airfoils at high angles of attack. This often limits the experimental capabilities of open-jet wind tunnels.

During the early 2000s, Devenport et al. [2,5] introduced the idea of hybrid anechoic wind tunnels (HAWTs), where the test volume is enclosed with tensioned Kevlar walls. The benefits of this configuration are rooted in the properties of the Kevlar cloth, as Kevlar is not only strong and highly durable, but was also proven to be acoustically transparent (i.e. has a low acoustic transmission loss) and it is near impermeable to flow [2] thanks to its very low $(2 \%-5 \%)$ open area ratio. The flow can also be separated from the microphones in hybrid anechoic wind tunnels, while HAWTs provide a very similar aerodynamic performance to hardwall wind tunnels. Aerodynamic corrections for HAWTs can be determined a-priori from the porosity and flexibility of the Kevlar walls using a low-order panel method [2, 6, 7].

The sound from the noise source of interest in both open-jet and HAWT configurations must propagate through the boundaries of the test section before it can reach the far field microphones. In the case of an open-jet configuration, the sound passes through a shear layer, while in HAWTs, it passes through a boundary layer and a Kevlar cloth. The propagating acoustic wave gets refracted and attenuated at the boundary of the test section. This effect is most commonly accounted for using the correction method of Amiet 8 that was originally developed for open-jet wind tunnels. Amiet's formulation corrects measured data for changes in both propagation time and pressure magnitude, providing a free field equivalent acoustic wind tunnel data. Recent works compared the effects of shear layer refraction in open-jet and HAWT wind tunnels [9 11]. It has been shown that the boundary layer and Kevlar wall alters the acoustic ray significantly less than a shear layer in the case of an open-jet wind tunnel. This is partially because the shear layer is much thicker than a boundary layer (assuming an identical test section to open-jet by simply enclosing it by Kevlar walls [11]), and because Kevlar deflects significantly less than the shear layer under

\footnotetext{
* Corresponding author:

Email address: m.szoke@vt.edu (Máté Szőke)
} 
lifting configurations within an open-jet wind tunnel. These observations manifest in dramatically reduced unsteady effects in the far field acoustic data, such as variability in signal magnitude and phase, loss of coherence, and variation in signal propagation time when compared to open-jet conditions [11].

While Amiet's shear layer correction model is a widely used aeroacoustic correction method, only a limited number of studies exist where its accuracy has been assessed for HAWT facilities [9, 10, 12]. The present work aims to experimentally measure the quantities determined by Amiet's shear layer correction model [8] in a HAWT configuration, namely, the acoustic ray propagation times and pressure magnitude corrections are assessed. Both of these metrics have partially been addressed in previous studies [9, 10, 12, but without a detailed uncertainty quantification. Also, it is still unknown how refraction through the boundary layer and Kevlar interface affects the frequency-energy content of the sound ray, which is also investigated in the present work.

To reach these goals, the acoustic signal of an impulse point source was captured experimentally in the acoustic far field using an array of microphones. The point source was generated using laser-induced plasma (LIP) in the Stability Wind Tunnel (SWT) of Virginia Tech (VT), which is a large-scale HAWT facility. While the acoustic measurement of LIP is relatively straightforward to perform, the acoustic properties of the LIP and the limitations of the instrumentation are important factors in such an investigation. The characteristics of the LIP and the experimental limits are therefore discussed in this paper prior to assessing the propagation characteristics of the LIP noise in a HAWT environment.

Laser-induced plasma is widely used across various scientific fields, such as in laser-induced breakdown spectroscopy [13], nondestructive security and military applications [14, room acoustics measurements [15], and mechanical vibration analysis [16, 17, to mention a few. Over the past two decades, it has been used for aeroacoustic studies as well. These works studied the pressure shielding effects of bodies [18, 19, shearlayer propagation effects [9, 11, 12, 20, and Kevlar transmission loss measurement 21, to mention a few. While some work has also been done in numerically and analytically modeling the noise source generated by LIP [22, 23], its fundamental properties (such as overall sound pressure level and pressure signature) are still challenging to determine by purely relying on the laser-optical system used to generate the plasma itself (laser energy, pulse width, focal distance, ambient conditions of air, etc). To gain an understanding of how the properties of the laser-optical system can be linked with the acoustic output of the LIP, some fundamentals of plasma dynamics must be understood.

Plasma can be generated using a focused laser beam such that a high enough energy density is reached per unit volume, namely on the order of $10^{9}-10^{11} \mathrm{~W} / \mathrm{m}^{3}$ [24, 25]. The focused laser beam creates an electric 
field in which the medium (air, in this case) ionizes, resulting in the production of free electrons and positive ions. The free electrons gain energy from the laser-electric field and this results in further ionization, or inverse Bremsstrahlung [13. It has been shown that the energy absorption rate of air increases with pulse interval (and also with energy per pulse) when the pulse interval is short enough for the electron density not to fully decay. The energy absorbed by the medium (air) was shown to saturate at approximately 60-70\% of the incident beam energy [15, 26, 27] with increasing laser power. At high-enough laser energy levels, the inverse Bremsstrahlung takes place even prior to the laser beam reaching the optical focal point. In this case, two overlapping plasma cores are formed, one at the optical focal point and another one slightly closer to the focusing optics [25, 26]. The resulting pressure wave is still a single pressure pulse (or monopole) because the development time of the plasma is still shorter than the time period of the generated acoustic pressure wave.

Various studies investigated $26-\sqrt[28]{28}$, through nanosecond Schlieren imaging, how plasma is formed in air at various laser energy levels. Further analyzing the high-speed Schlieren images using von NeumannTaylor's point strong explosion theory [29, 30] revealed that the pressure wave generated by the LIP initially propagates at supersonic speeds, on the order of 4-6 km/s [26]. From an aeroacoustic point of view, this can be considered as a source of discrepancy between the assumed (or modeled) and actual (measured) arrival time of acoustic rays from the source to the far field observers. This discrepancy has previously been neglected in LIP studies [9, 10, 31 as it can only be accounted for by performing microsecond Schlieren imaging of the LIP. From these images, the supersonic propagation speed and its time duration can be determined. Such an experiment and analysis are presented in this paper to assess the discrepancy of acoustic propagation times.

This paper investigates the unsteady propagation characteristics of sound waves generated by LIP in a HAWT environment and provides a detailed uncertainty analysis of the acoustic investigation performed. This is in support of better understanding the limitations of commonly used acoustic measurement chains, and to accurately quantify acoustic pressure wave arrival times and pressure correction magnitudes as proposed by Amiet's shear layer correction model [8]. To reach these goals, the paper first discusses the experimental approach used for this study in Section 2 Section 3 then provides a detailed discussion of the metrics used in this study. Section 4 provides a quantitative analysis and a detailed discussion of the results with the major findings summarized in Section 5. 


\section{Experimental Approach}

\subsection{The Virginia Tech Stability Wind Tunnel}

The Stability Wind Tunnel (SWT) is a low-speed closed-circuit wind tunnel used for a mix of commercial testing, research, and educational experiments on the campus of Virginia Tech in Blacksburg, Virginia [2, 6]. The facility, driven by a $600 \mathrm{HP}$ fan, can generate speeds of up to $80 \mathrm{~m} / \mathrm{s}$ through its $7.3 \mathrm{~m} \mathrm{long}, 1.85 \mathrm{~m}$ square test section. Flow through the empty test section is characterized [2, 6] by very low turbulence intensity, increasing from $0.021 \%$ at $21 \mathrm{~m} / \mathrm{s}$ to $0.031 \%$ at $57 \mathrm{~m} / \mathrm{s}$.

Two test section configurations are available. The hard-wall "aerodynamic" test section configuration has walls formed from a sequence of aluminum panels. The Kevlar-walled aeroacoustic test section configuration used in the present work replicates the same interior dimensions as the hard wall test section configuration. The side walls of this test section, however, include $4.2 \mathrm{~m}$ long openings that span the full $1.85 \mathrm{~m}$ test section height. These openings are covered using tensioned Kevlar cloths that are almost transparent to sound and only slightly porous to the flow. The sound generated by models in the test section travels out through these acoustic windows into the anechoic chambers that flank the test section. Most acoustic measurements are made using microphones placed in these chambers. Kevlar 49, Style 120 cloth is used for the windows, which is a scrim made from a plain weave of Kevlar 49 fiber with 13.4 threads per centimeter in both warp and weft directions. For the tests reported here, cloth provided by EAS Fiberglass Company was used with an open area ratio of $1.8 \%$, weight of $58 \mathrm{~g} / \mathrm{m}^{2}$, thickness of $0.08 \mathrm{~mm}$, and equivalent membrane thickness of $0.021 \mathrm{~mm}$. The Kevlar forming the acoustic windows is supported on tensioning frames under a nominal no-flow tension of $1500 \mathrm{~N} / \mathrm{m}$. The ceiling and floor of the acoustic test section are formed, primarily, from perforated metal panels backed by $0.46 \mathrm{~m}$ acoustic wedges to serve as sound absorbers. The perforated panels are covered with additional Kevlar cloth in order to form a low-noise flow surface.

\subsection{Aeroacoustic Experiments at the Stability Wind Tunnel}

A Quantel Evergreen Nd:YAG double pulsed Q-switched laser was used to create a laser beam whose nominal maximum energy content is $200 \mathrm{~mJ} /$ pulse at a $532 \mathrm{~nm}$ wavelength and is characterized with a pulse width of 8 to $10 \mathrm{~ns}$. The nominal pulse-to-pulse energy stability of the laser system is $3 \%$ rms. Figure 1 depicts the optical arrangement used to generate the LIP. The beam is focused into a point using a series of lenses, namely an $f_{1}=-50 \mathrm{~mm}$ concave, and two $f_{2}=f_{3}=1200 \mathrm{~mm}$ convex lenses. The LIP is generated at a distance of $1200 \mathrm{~mm}$ from the second convex lens. The LIP is positioned at the center of the empty SWT test volume with the optical axis of the system fed through an optical window mounted to the test 
section floor, see Fig. 2. This optical access is provided using a $6 \mathrm{~mm}$ thick glass with low iron content to minimize the energy loss of the beam as it passes through the window. The repetition rate of the laser was set to 5 pulses per second, which was found to result in the formulation of LIP for $95 \%$ of the pulses. A Menlo System APD210 type photodetector was used to capture the instant of occurrence of the laser beam pulses. From an acoustic point of view, it is assumed that this time instant is identical to the formation of the acoustic pulse generated by the LIP. The free stream velocity in the test section $\left(u_{\infty}\right)$ was initially set to $0 \mathrm{~m} / \mathrm{s}(M=0)$ then was varied between $20 \mathrm{~m} / \mathrm{s}$ and $60 \mathrm{~m} / \mathrm{s}$ in steps of $10 \mathrm{~m} / \mathrm{s}$, for which the corresponding Mach numbers $(M)$ were $0.058,0.087,0.116,0.144$ and 0.173 , respectively.

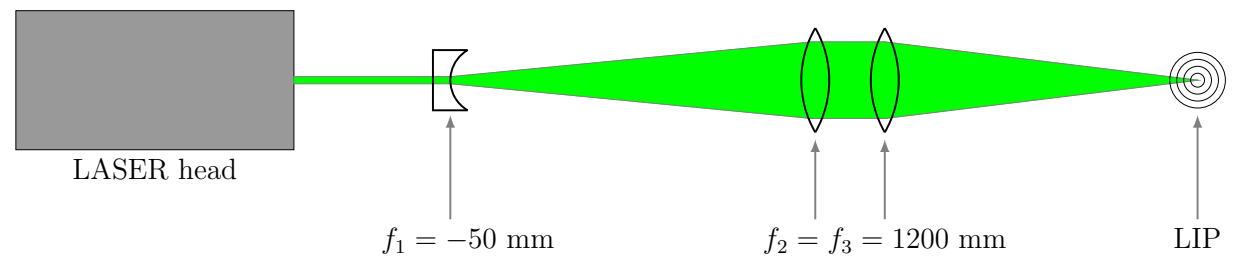

Figure 1: The schematics of the laser-optical assembly used in this study.

The acoustic signal in the far field (i.e. starboard-side anechoic chamber) was captured using a 251element beamforming array that consists of phase-matched 1/4 inch $(6.35 \mathrm{~mm})$ G.R.A.S. 40PH-S5 type microphones. The microphones have a flat frequency response $( \pm 2 \mathrm{~dB})$ over the frequency range of $5 \mathrm{kHz}$ to $20 \mathrm{kHz}$. The microphone array, built by AVEC Inc., has four interlocking spiral-shaped subarrays and it spans over the entire area of the Kevlar wall (i.e. $4.2 \mathrm{~m} \times 1.85 \mathrm{~m}$ ), see Fig. 2 This configuration offers a good spatial resolution at both low and high frequencies, a wide range of potential observer angles, and high modularity for various potential wind tunnel test cases. Signals from all sensors (microphones and photodetector) were anti-alias filtered using an in-house analog low-pass filter characterized with a $20 \mathrm{kHz}$ corner frequency. The signals were then recorded simultaneously at $f_{s}=192 \mathrm{kSamples} / \mathrm{s}$ for a time span of 32 seconds using four PCIe-16AI64SSC 16-bit analog to digital converters manufactured by General Standards Corporations. With these measurement settings, a maximum of 160 pulses were acquired at each flow speed. Also, the fine temporal resolution of the acoustic field provides an approximately $1.8 \mathrm{~mm}$ spatial resolution of the sound waves. The details of measurement chains in aeroacoustic facilities usually differ from organization to organization, but the overall test specifications are often similar, therefore, the investigation presented here is expected to provide results that are generally applicable over a typical range of instrumentation selection 2, 11, 32. 


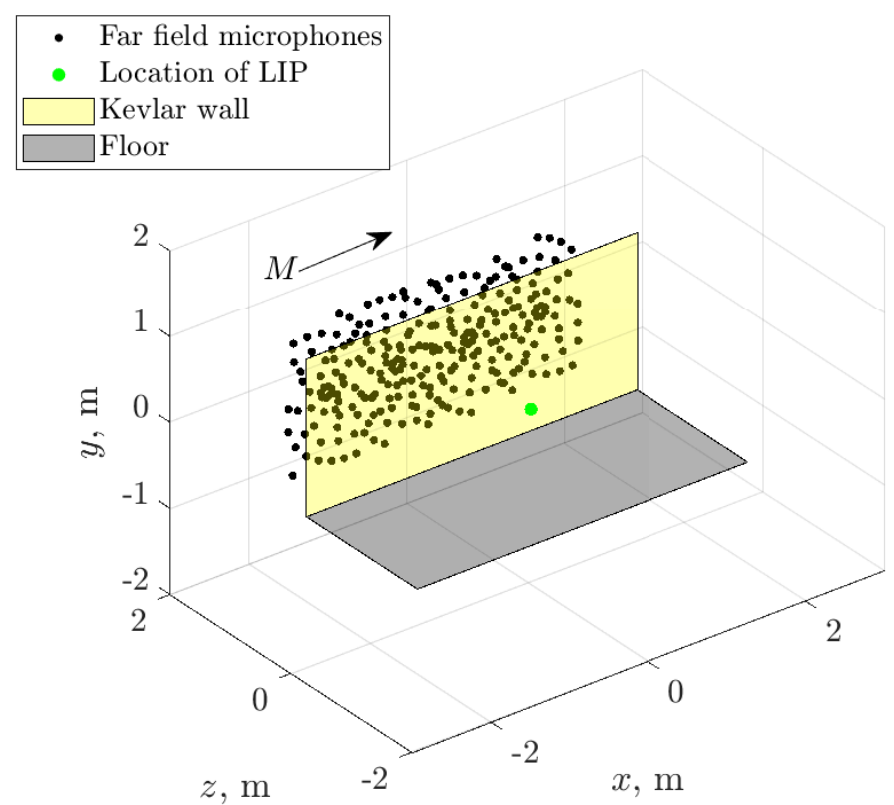

Figure 2: Schematics of the measurement setup within the SWT: far field microphone locations and the location of LIP in the test section. The free stream velocity is in the positive $x$ direction.

\subsection{Schlieren Photography}

To capture the shape of the plasma and to visualize (i.e. track) the propagation of the shockwave generated by the LIP, a high-speed Schlieren imaging of the LIP was performed. The system, whose schematic is shown in Fig. 3, consisted of an ABET Technologies light source (model LS-180-Xe), two achromatic lenses with a focal length of $f=300 \mathrm{~mm}$ with a diameter of 2-inches, and a razor blade (as a knife-edge). A Phantom v2512 type high-speed camera with an $f=100 \mathrm{~mm} / \mathrm{F} 2.8$ Tokina macro lens was used to capture the Schlieren images. The camera was operated at a resolution of $256 \times 256$ pixels enabling a $200 \mathrm{kFrames} / \mathrm{s}$ frame rate and a 1.62 microsecond exposure time per frame. The optical setup was calibrated prior to the experiments from which the calibration constant of $17.6 \mathrm{pixel} / \mathrm{mm}$ was found, therefore, the field of view was $14.5 \times 14.5 \mathrm{~mm}$ in size. The optical axes of the Schlieren setup and the laser were perpendicular to each other, as noted in Fig. 3. While the frame rate of the camera was too low to capture the formation of the plasma core itself, this sampling rate was near identical to the acoustic sampling rate $(192 \mathrm{kSamples} / \mathrm{s})$, therefore it allowed the visualization of the shockwave around the plasma. This enables the determination of shockwave propagation speed from the Schlieren images. The video recording was triggered by the Q-switch signal of the laser module to ensure that the first captured frame corresponds to the laser emission time. 


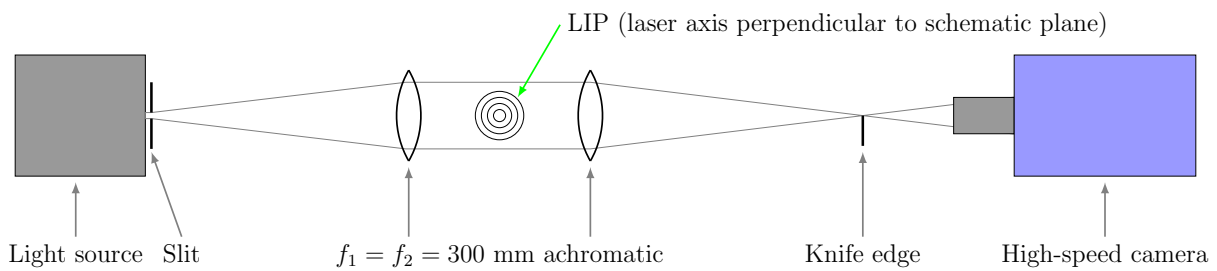

Figure 3: Schematics of the high-speed Schlieren imaging setup.

\section{Data Processing Approach}

\subsection{Modeled Arrival Time}

The shear layer correction method proposed by Amiet [8] was applied for the three-dimensional test volume. In Fig. 4 the schematic of Amiet's correction method is depicted for a simplified two-dimensional case. According to the model, the sound emitted from the source $(\mathrm{S})$ propagates along $r_{1}$ within the test section $\left(u_{\infty}>0\right)$ until refraction occurs at the Kevlar wall $(\mathrm{P})$ followed by propagation along $r_{2}$ until the sound reaches the far field microphones $(\mathrm{O})$. The displacement of the Kevlar wall (point $\mathrm{P}$ ) is neglected because the SWT test section was empty during the experiments. In the absence of flow, the sound propagates along $r_{m}$. The polar and azimuth angles $\left(\theta, \psi\right.$, respectively), $r_{1}$, and $r_{2}$ are found based on Bahr et al. 9. From this, the time required for the sound to propagate along $r_{1}$ and $r_{2}$ can be obtained using the reference [9], and it is denoted as $\tau_{\text {Amiet }}$.

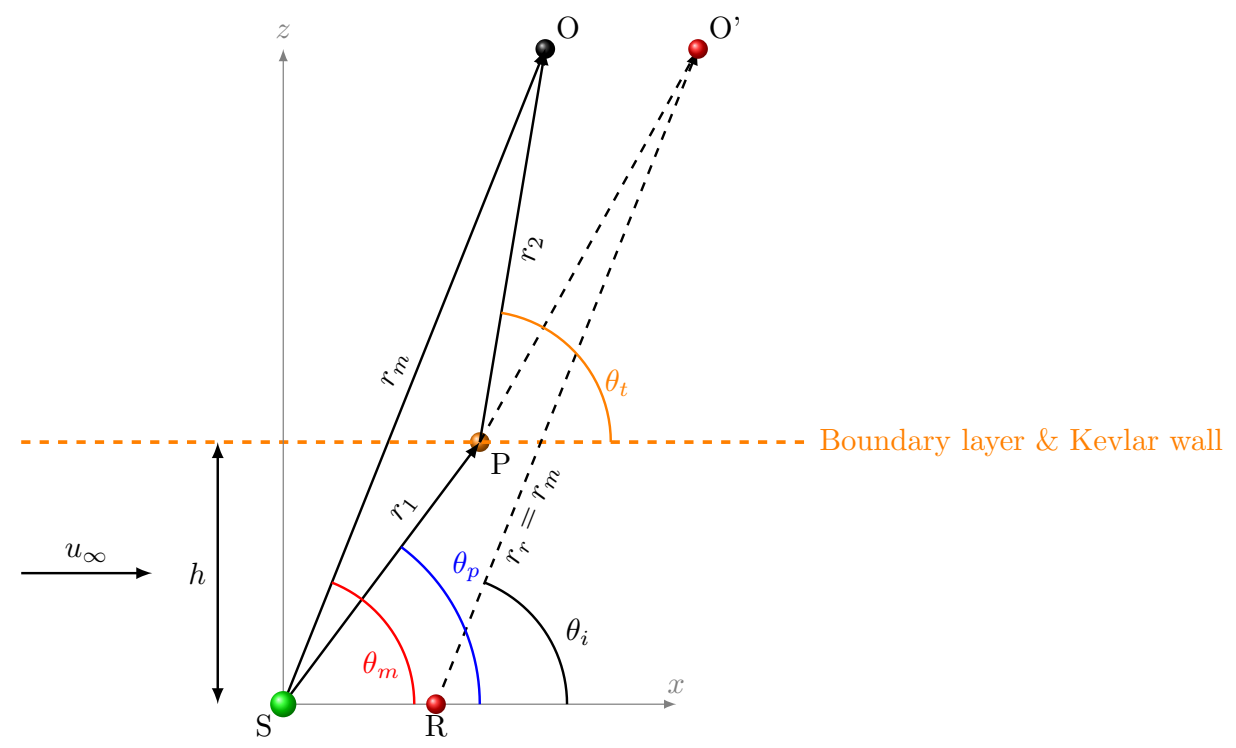

Figure 4: The acoustic ray path as it passes through the boundary layer and Kevlar wall. 

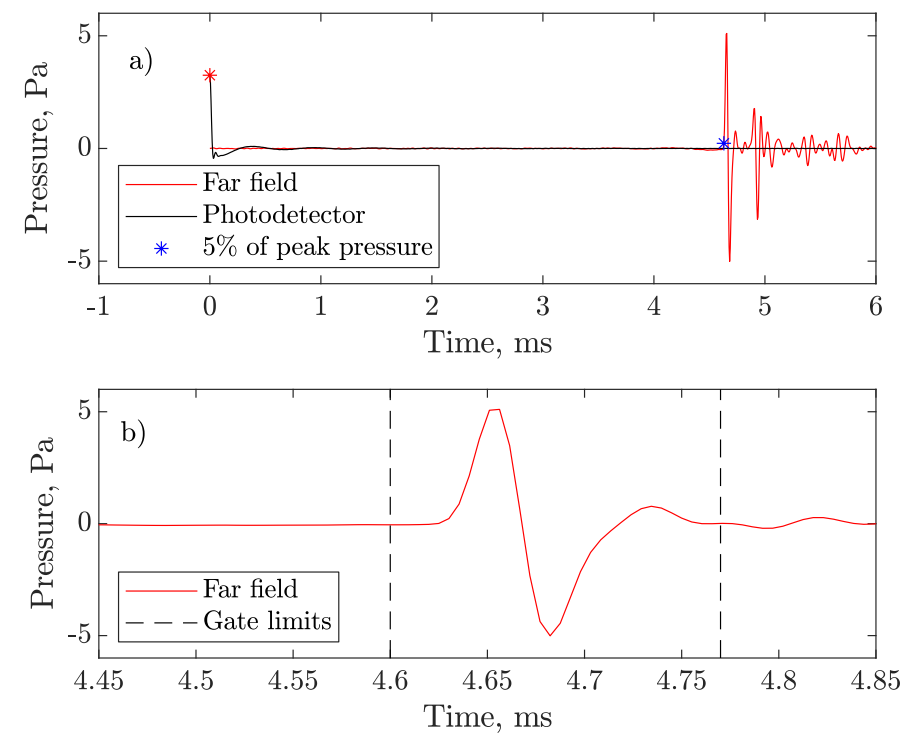

Figure 5: The determination of arrival time (a) and obtaining the energy level of the acoustic pressure (b).

\subsection{Measured Arrival Time}

To analyze the data in the time domain, the pressure signal obtained from the far field microphones is first high-pass filtered using a minimum-order finite impulse response filter where the signal is compensated for any delay introduced during the filtering process. In particular, Matlab's highpass digital filter was used for filtering at an $f_{H P}=1 \mathrm{kHz}$ corner frequency [33]. The digital filter helps to isolate the sound of the LIP in the time domain, as the background noise of the SWT is mainly limited below this frequency. The maximum energy content of the LIP in the frequency domain was observed well above $10 \mathrm{kHz}$ by previous studies [11, 22, therefore, the frequency content of the LIP is well separated from the corner frequency of the high-pass filter $(1 \mathrm{kHz})$. For this reason, the digital filter is expected to have a negligible effect on the signal of the LIP.

After high-pass filtering, the arrival time, $\tau_{a}$, of the pressure signature is determined as follows: (1) the photodetector signal is used to identify the time instant of laser light emissions, from which the time signal of the microphones are subdivided into blocks, such that the laser light emission happens at the beginning of each block ( $t=0 \mathrm{~s}$, see Fig. 5(a)); (2) the arrival time within each block is defined as the instant at which the pressure signal surpasses $5 \%$ of the peak magnitude; (3) the mean value of arrival times, $\tau_{a}$, is then determined by ensemble averaging the arrival times for all blocks with plasma formations.

From this, an error variable $(\varepsilon)$ is defined which quantifies the discrepancy between the measured $\left(\tau_{a}\right)$ and calculated $\left(\tau_{\text {Amiet }}\right)$ arrival times. It is interesting to note that the difference between $\tau_{a}$ and $\tau_{\text {Amiet }}$ 
may be used to assess microphone location accuracy. However, to minimize the influence of uncertainty in the microphone locations, the change in propagation time from flow to quiescent conditions is compared between the measured data and Amiet's model. In a rough sense, this metric is near-independent of the microphone installation uncertainty for the measured propagation times. It is defined as

$$
\varepsilon_{\tau}=\left(\left.\tau_{a}\right|_{M>0}-\left.\tau_{a}\right|_{M=0}\right)-\left(\left.\tau_{\text {Amiet }}\right|_{M>0}-\left.\tau_{\text {Amiet }}\right|_{M=0}\right)
$$

where $M=0$ and $M>0$ refers to cases when the flow speed in the wind tunnel is zero and nonzero, respectively. In the current work, $\varepsilon_{\tau}$ remains dimensional as normalizing with $\left(\left.\tau_{\text {Amiet }}\right|_{M>0}-\left.\tau_{A m i e t}\right|_{M=0}\right)$ to compute a relative error results in dividing by near zero values at $\theta \approx 90^{\circ}$.

\subsubsection{Modeled Pressure Correction}

The shear layer correction model proposed by Amiet [8] also offers the possibility to correct the pressure magnitude to a free field equivalent value. In a HAWT environment, like in the SWT, the sound passes through a turbulent boundary layer and a tensioned Kevlar fabric window prior to reaching the far field microphones. Amiet's model assumes the presence of uniform parallel flow, and does not allow for the incorporation of any Kevlar transmission loss or turbulent scattering. However, as the Amiet correction is a pressure ratio, if the Kevlar's transmission coefficient is approximately flow speed-independent and changes in incidence angle are small, it should divide out in this ratio. Turbulence effects must still be neglected. In this work, Amiet's corrections are computed on an equal-emission distance basis, $r_{r}=r_{m}[9$.

As was shown by Rossignol et al. 22, the far field sound pressure of a LIP sound source must be corrected for Doppler effect. This correction can be approximated by multiplying Amiet's pressure ratio with

$$
\frac{\beta^{2}}{1-M x_{P} / \sigma_{c}}
$$

where $\beta^{2}=\left(1-M^{2}\right), \sigma_{c}=\left[x_{P}^{2}+\beta^{2}\left(y_{P}^{2}+z_{P}^{2}\right)\right]^{1 / 2}$ and $\left(x_{P}, y_{P}, z_{P}\right)$ are the coordinates of the refraction point P, see Fig. 4 .

\subsubsection{Measured Pressure Correction}

The current measurement configuration enables the direct measurement of the theoretical pressure correction defined by Amiet. To do this, the pressure signature of the LIP measured in the far field needs to be gated on each microphone signal for each block of data, see Fig. 5(b). Gating is required as Amiet's corrections only account for the direct path of sound propagation. To mitigate the uncertainty caused by 
the pulse-to-pulse energy stability of the laser-emission pulses, the acoustic signals are normalized using the peak pressure value detected within the block of a dedicated far field microphone located at a $\theta_{m} \approx 90^{\circ}$ observer angle. Once the initial pulse is isolated $\left(p_{b l o c k, i}(t)\right)$, each block is squared and integrated to yield a block energy level, see Fig. 5(b). The square root of this level both without $\left(\hat{p}_{0}\right)$ and with flow $\left(\hat{p}_{M}\right)$ can be defined as

$$
\left|\frac{\hat{p}_{0}}{\hat{p}_{M}}\right| \text {. }
$$

As $r_{r}$ is defined as the equivalent measurement position without flow for a flow-based measurement, this quantity is already on an $r_{r}=r_{m}$ basis. It is important to note that, as with Amiet's method, neither $\hat{p}_{0}$ nor $\hat{p}_{M}$ is corrected for the pressure attenuation caused by the Kevlar cloth. Under the same assumptions as the discussion for Amiet's correction, the influence of the Kevlar is expected to approximately cancel in the current expression. From this, another error variable $(\varepsilon)$ can be defined which quantifies the discrepancy between the measured pressure correction and the theoretical pressure correction and it is defined as

$$
\varepsilon_{p}=20 \log _{10}\left|\frac{\hat{p}_{0}}{\hat{p}_{M}}\right|-20 \log _{10}\left|\frac{\hat{p}_{\text {corr }}}{\hat{p}_{\text {meas }}}\right|_{\text {Amiet }} .
$$

Considering Eq. (4), the pressure correction ratio $\left(\left|\hat{p}_{0} / \hat{p}_{M}\right|\right)$ is integrated using time-domain data and therefore no information is available on its frequency dependence. However, the Fourier transform of the gated signal, $p_{b l o c k, i}(t)$, (see Fig. 5(b)) enables the investigation of the pressure correction ratio in the frequency domain. To reach this goal, the gated signal was zero padded and centered within a signal corresponding to a temporal length of 100 milliseconds. The fast Fourier transform of the zero padded signal was then performed. After multiplying the Fourier-transformed data with its complex conjugate, an identical variable to that of $\hat{p}$ is obtained (see Eq. (4) but with carrying frequency-dependent information. The resulting quantities are denoted as $P_{0}(f)$ and $P_{M}(f)$, corresponding to the without flow $(M=0)$ and with flow $(M>0)$ cases, respectively. From this, the frequency-dependent discrepancy $\left(E_{p}(f)\right)$ between the frequency dependent measured pressure correction $\left(\left|P_{0}(f) / P_{M}(f)\right|\right)$ and the frequency independent theoretical pressure correction $\left(\left|\hat{p}_{\text {corr }} / \hat{p}_{\text {meas }}\right|_{\text {Amiet }}\right)$ can be defined as

$$
E_{p}(f)=20 \log _{10}\left|\frac{P_{0}(f)}{P_{M}(f)}\right|-20 \log _{10}\left|\frac{\hat{p}_{\text {corr }}}{\hat{p}_{\text {meas }}}\right|_{\text {Amiet }} .
$$




\subsection{Measured Shockwave Speed}

Similarly to the studies of Leela et al. [26], the propagation speed of the shockwave generated by the LIP is determined using high-speed Schlieren photography. The location of the shockwave was visually determined for using the Schlieren images and, via the spatial calibration constant and the known time difference $(5 \mu \mathrm{s})$ between the frames, the shockwave speed was calculated.

\subsection{Modeled Shockwave Speed}

The von Neumann-Taylor's point strong explosion theory [29, 30] enables the calculation of shockwave radius (measured from the center of the explosion) by knowing the energy released into the small volume of fluid (air, in this case) and can be written as

$$
R_{s w}=C\left[\frac{E_{s} t^{2}}{\varrho}\right]^{\frac{1}{5}}
$$

where $E_{s}$ is the energy absorbed by the air from the incident laser beam, $t$ is the time elapsed from the instant of the laser emission, and $\varrho$ is the density of air prior to the laser emission time (undisturbed ambient air density), while $C$ is a constant purely dependent on the ratio of specific heat $(\gamma)$ of air and it can be written as

$$
C=\left[\frac{75(\gamma-1)(\gamma+1)^{2}}{16 \pi(3 \gamma-1)}\right]^{\frac{1}{5}}
$$

The energy absorbed by air from the laser emission is approximated using the results of Leela et al. [26], who showed that the absorbed laser energy saturates with increasing beam energy at approximately $60-80 \%$ of the incoming laser beam energy. Due to the age of the laser system used in this study, the lower end of the absorption coefficient reported by Leela et al. [26] was considered, i.e., $E_{s}=0.6 E_{L}$ was used, with $E_{L}=200 \mathrm{~mJ}$ per pulse.

The shockwave speed can simply be determined as the rate of change of shockwave radius $\left(R_{s w}\right)$

$$
V_{s w}=\frac{\mathrm{d} R_{s w}}{\mathrm{~d} t}=\frac{2 C}{5}\left[\frac{E_{s}}{\varrho t^{3}}\right]^{\frac{1}{5}}
$$

The model assumes that the shockwave speed decays exponentially with time, which in the current case is valid until it reaches the speed of sound, $c_{0}=\sqrt{\gamma R T}$, where $R$ is the specific gas constant of air and $T$ is the ambient temperature measured in Kelvin. Setting these two quantities equal determines the time instant $\left(t^{*}\right)$ at which the shockwave surpasses the speed of sound, i.e. $V_{s w}\left(t=t^{*}\right)=c_{0}$. This information can be 
used to answer the question of how much faster does the shockwave travel over a certain period of time as opposed to a soundwave originated from the same physical location, or in other words, how much faster does the sound of LIP reaches the observer as opposed to a sound wave originated from the same physical location, as it is assumed in Amiet's model. This discrepancy is the difference between the time integral of the sound wave propagation speed and the time-integral of the shockwave propagation speed.

\section{Results}

This section presents the results from the Schlieren experiments and the aeroacoustic experiments. To obtain an understanding of the LIP behavior and experimental limits, the uncertainty analysis of the acoustic data is presented first, followed by the analysis of the Schlieren images. The analysis of these data sheds light on the limitations on the experimentally obtained arrival times and pressure correction parameters. Once an understanding on the limitations of the experiments and the metrics used to assess the properties of shear-layer refraction is gained, the arrival time of the pressure pulses generated by the LIP and the pressure correction values are presented and analyzed.

\subsection{Uncertainty analysis}

The microphones used in this study have a flat frequency response up to $20 \mathrm{kHz}$ and the sampling rate used to acquire the acoustic signal of the LIP was set to the maximum available rate $\left(f_{s}=192 \mathrm{kSamples} / \mathrm{s}\right)$. The time span of the LIP is approximately $0.1 \mathrm{~ms}$, corresponding to approximately $10 \mathrm{kHz}$ (see Fig. 5), therefore, the settings of the experimental chain were at the limit of the instrumentation. For this reason, it is important to understand how the experimental chain performed under these conditions. From the repeatedly acquired LIP pressure signals in the aeroacoustic experiments, the uncertainty of the arrival times $\left(\tau_{a}\right)$ and sound levels $\left(20 \log _{10}\left(\hat{p}_{M} / p_{0}\right)\right)$ can be addressed. These parameters can shed light on the accuracy of the aeroacoustic analysis presented here alongside with the measurement limitations of the instrumentation used in this study. Also, the change in the experimental uncertainty as a function of flow speed can help to understand the in-flow behavior of the LIP. The uncertainty analysis presented here follows the standard error of the mean analysis at a confidence interval of $95 \%$ 34 with $\delta_{\xi}=1.96 \operatorname{std}\left(\xi_{i}\right)$ representing the uncertainty of variable $\xi_{i}$ about its expected mean value.

The uncertainty of the arrival times as a function of Mach number is presented in Fig. 6, where the dashed line indicates the time step of the data acquisition system. The data shown in Fig. 6 was ensembleaveraged for all blocks of LIP formulation, and the resulting individual microphone-specific uncertainties 


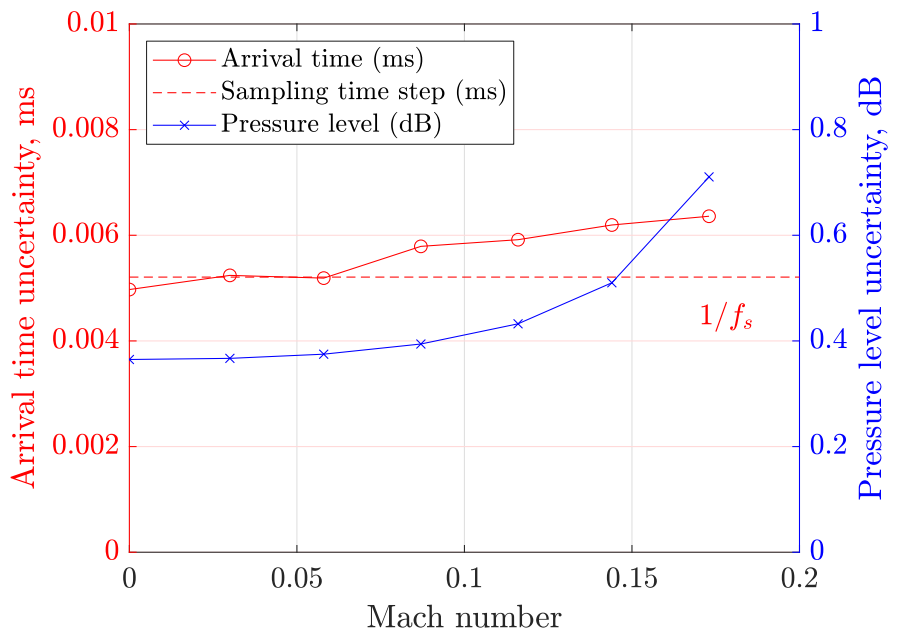

Figure 6: Uncertainties of the arrival times and pressure levels as a function of Mach number.

were also averaged across all 251 microphones. The results are near-independent of the flow speed because even at $M=0.173$, the uncertainty remains in the vicinity of the data acquisition time step (0.0052 ms). This suggests that the temporal uncertainty is bounded by the sampling limit of the data acquisition system. This is in agreement with previous studies [11, where for most of the conditions investigated in a HAWT environment, the standard deviation of arrival times $\left(\operatorname{std}\left(\tau_{a}\right)\right)$ was found to remain below the sampling rate used in this study.

The uncertainty of the pressure correction discrepancy $\left(\varepsilon_{p}\right)$, on the other hand, shows a stronger dependence on the flow speed, see Fig. 6. At low speeds, $M<0.1$, the uncertainty remains below $0.5 \mathrm{~dB}$, which is most likely determined by the microphones. However, as the flow speed increases, the uncertainty of the pressure levels departs from this instrumentation-related limit. To gain an insight into the source of this discrepancy, Fig. 7 presents the uncertainty of the pressure levels at each microphone location as a function of flow speed at $M=0$ and 0.173. In Fig. 7, the flow direction is defined left to right, while the LIP is located at the origin of the coordinate system. As suggested by Fig. 7 , the experimental uncertainty has a uniform distribution at $M=0$ among the microphone locations, and therefore, it is dominated by the instrumentation used in this study. However, at $M>0.1$, the uncertainty distribution changes. At $M=0.173$, a higher uncertainty is observed at both upstream and downstream emission polar angles $\left(\theta_{i}>120^{\circ}\right.$ and $\left.\theta_{i}<60^{\circ}\right)$. The emission azimuth angle $\left(\psi_{i}\right)$ is observed to have a negligible effect on the distribution of uncertainty. These observations suggest an interaction between the acoustic signature of the LIP and the flow field, presumably this interaction is most significant when the acoustic ray passes through the boundary layer and the Kevlar wall at a shallow angle. 

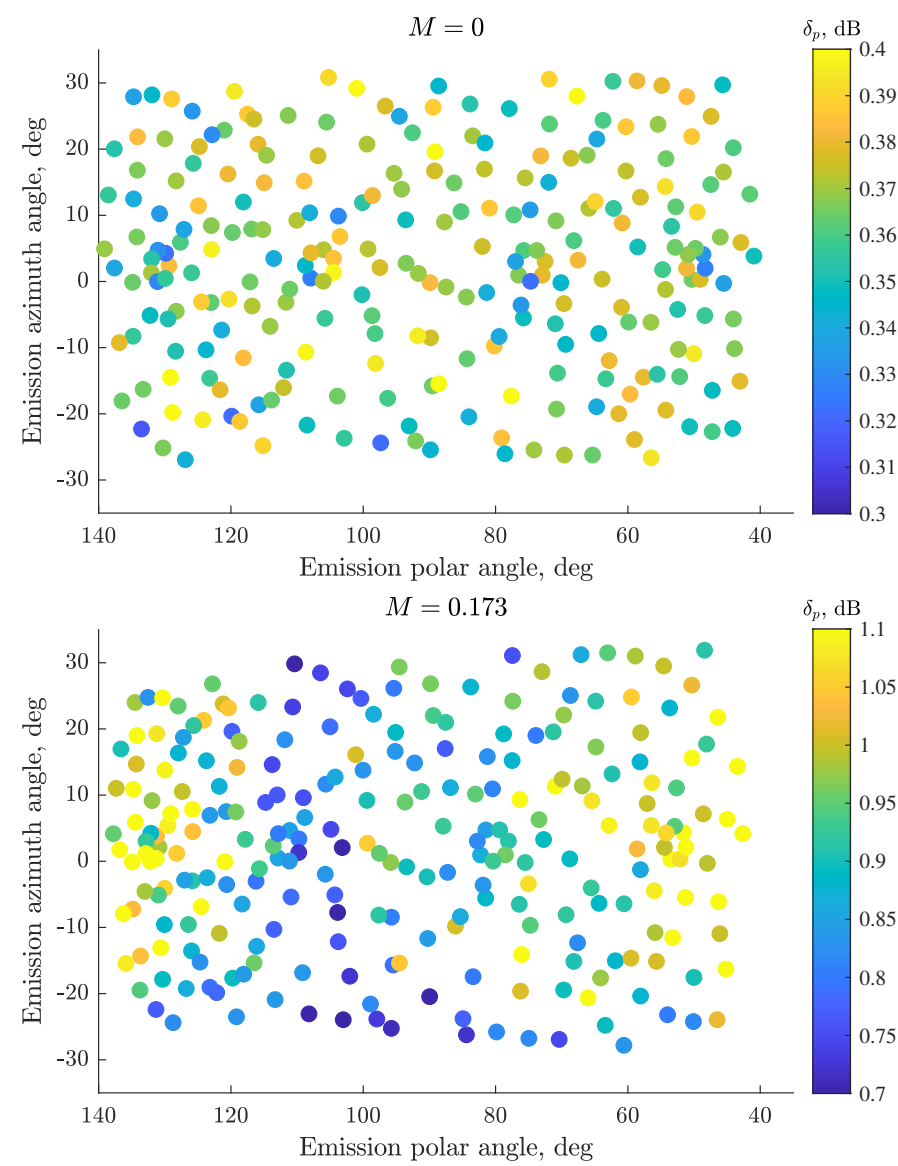

Figure 7: Pressure level uncertainty of individual far field microphones as a function of emission polar and azimuth angles at $M=0$ and $M=0.173$. 


\subsection{Shockwave Speed Analysis}

The shockwave generated by the LIP has a short time period when the generated pressure wave travels at a supersonic wave speed [10, 13, 26, 27. When comparing the arrival times of the LIP-generated sound waves to Amiet's model of shear layer correction, the initial supersonic wave speed of LIP results in an increase in the arrival time discrepancy of the pressure pulses $\left(\varepsilon_{\tau}\right)$. For this reason, the high-speed Schlieren images are used in this study to determine the radius and also the wave speed of the shockwave at certain time instants. Also, von Neumann-Taylor's point strong explosion theory is used to model the shockwave propagation speed as a function of time.

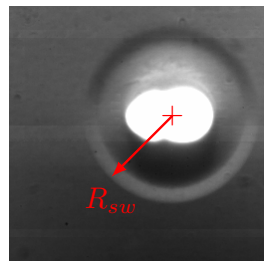

a) $t=5 \mu \mathrm{s}$

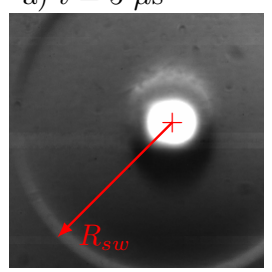

c) $t=15 \mu \mathrm{s}$

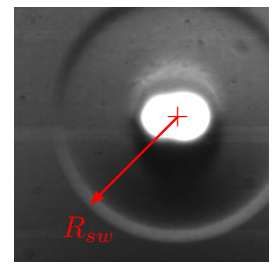

b) $t=10 \mu \mathrm{s}$

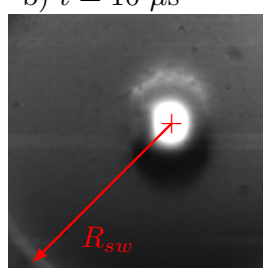

d) $t=20 \mu \mathrm{s}$

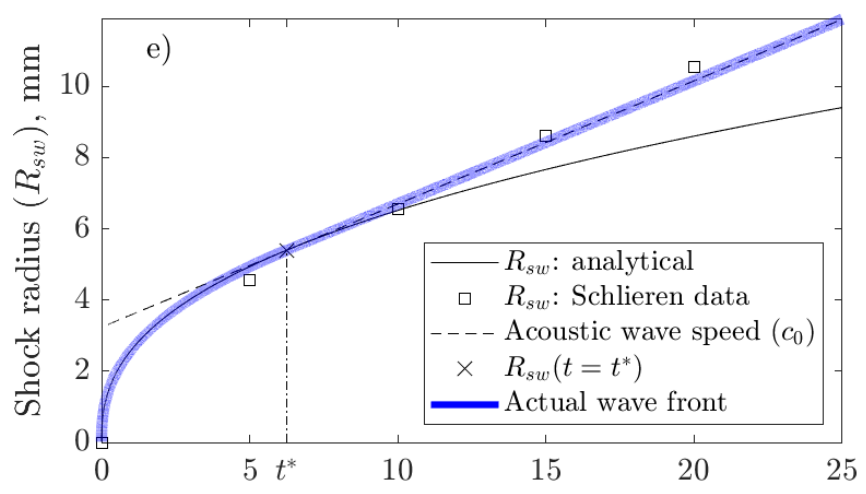

Time, $\mu \mathrm{s}$

Figure 8: High-speed Schlieren images of the laser-induced plasma (a-d) and the measured and calculated radius of the shockwave as a function of time (e). The direction of the laser beam is defined from right to left in the Schlieren images.

Figure 8 (a-d) presents the high-speed Schlieren images at four time instants measured from the laser emission time $(t=0 \mathrm{~s})$. The generated shockwave can be observed at each time instant with its center and radius $\left(R_{s w}\right)$ marked by a red cross and arrow, respectively. At $t=0 \mathrm{~s}$, the presence of the laser beam resulted in the overexposure of the frame, hence that frame carries no valuable information and it is not presented here. The direction of the laser beam is defined from right to left in the Schlieren images. As was reported previously [25, 26], at lower laser energy levels $(50 \mathrm{~mJ})$, the generated plasma is spherical in shape, while at higher laser energy levels $(>150 \mathrm{~mJ})$, the plasma has an elongated, pear-like shape. In agreement with this, the shape of the plasma observed in Fig. 8(a) has a pear shape at $t=5 \mu \mathrm{s}$ as the power level of the laser was set to $200 \mathrm{~mJ}$ in this study. The plasma is slightly larger on its right half, i.e. on the side being closer to the laser-optical system, which is caused by inverse Bremsstrahlung [26 28].

Figure $8(\mathrm{e})$ presents the measured shockwave radius (labeled as $R_{s w}$ : Schlieren data) as a function of time alongside with the theoretical shock radius determined using von Neumann-Taylor's point strong explosion 
theory (labeled as $R_{s w}$ : analytical). The time instant at which the model predicts that the shockwave speed passes the acoustic wave propagation speed $\left(c_{0}\right)$ is marked as $t^{*}$. At this time instant, a tangent is drawn to the modeled shockwave radius with its gradient being equal to the acoustic wave speed (labeled as acoustic wave speed). The high-speed Schlieren data confirms that for a short period of time $\left(t<t^{*}\right)$ after the laser emission, the shockwave propagates at a supersonic speed, therefore, until this time instant, the shockwave radius follows von Neumann-Taylor's point strong explosion theory. For $t>t^{*}$, the results show that the shockwave propagation speed is equal to the acoustic wave speed $\left(c_{0}\right)$. This observation is denoted using the blue curve in Fig. 8(e), where the actual wave front radius follows $R_{s w}$ as predicted by von Neumann-Taylor's point strong explosion theory when $t<t^{*}$, and then the wave propagates with the speed of sound for $t>t^{*}$.

The sound wave generated by the LIP reaches a certain observer location over a shorter period of time (or propagates farther over a constant time window) than a theoretical impulse monopole source emitted from the same location and time instant $(t=0 \mathrm{~s})$ would. This difference is observed as a discrepancy in $\varepsilon_{\tau}$ because Amiet's model of shear layer refraction assumes a sonic propagation wave speed. The data obtained from the high-speed Schlieren images can be used to determine this discrepancy. Based on the experimental data presented in Fig. 8, the discrepancy was found to be 0.011 millisecond, which is equivalent to a distance of $3.67 \mathrm{~mm}$ when multiplied with $c_{0}$, while using von Neumann-Taylor's model, the discrepancy was found to be $0.0094 \mathrm{~ms}$, or $3.24 \mathrm{~mm}$. In comparison with the sampling rate of the acoustic measurements used in this study $\left(1 / f_{s}=0.0052\right.$ millisecond $)$, this discrepancy can be considered non-negligible as it is equivalent to approximately two time samples in the time domain of the acoustic data. The presented high-speed Schlieren experiment was performed at quiescent conditions $(M=0)$, therefore the findings presented here are valid primarily for the no flow case. A rather limited number of studies are available on the effect of crossflow on the plasma dynamics of LIP, therefore more investigations using nanosecond Schlieren imaging would be necessary to understand the effects of crossflow on the acoustic signature of LIP.

\subsection{Arrival Times}

Figure 10 presents the error of the arrival times $\left(\varepsilon_{\tau}\right)$ obtained from measurements conducted in the Stability Wind Tunnel for a range of Mach numbers. The definition of $\varepsilon_{\tau}$ is given in Eq. (1). Results are shown as a function of emission polar angle $\left(\theta_{i}\right)$ and emission azimuth angle $\left(\psi_{i}\right)$, see Fig. 4, where the Mach vector (flow direction) is defined from left to right.

The lowest discrepancy was observed at $\theta_{i}=90^{\circ}$ and $\psi_{i}=0^{\circ}$ for all conditions under investigation. The discrepancy is observed to increase when departing from this location with the increase mainly dominated 
by the emission polar angle and a less significant dependence is observed on the emission azimuth angles. The results presented in Fig. 10 are always positive $\left(\varepsilon_{\tau}>0\right)$, therefore Amiet's model underestimates the arrival time. While the high-speed Schlieren imaging revealed that the LIP-generated sound wave reaches the observer in a shorter period of time than an ideal source would, this effect should cancel in Eq. (1) assuming that the initial supersonic propagation time is not affected by the crossflow. This suggests that the crossflow may affect the acoustic signature of the LIP.

The increase in Mach number further increases the arrival time discrepancy. This suggests that the presence of the flow (and its effects) might be used to explain the source of the positive discrepancy. Amiet's model of shear layer refraction assumes a simplified model of the flow, where the refraction takes place over an infinitely thin layer (shear layer) with this interface considered as a plane. In addition, the flow in the model is uniform across the test volume and the flow speed is independent of the streamwise coordinate (or polar angles).

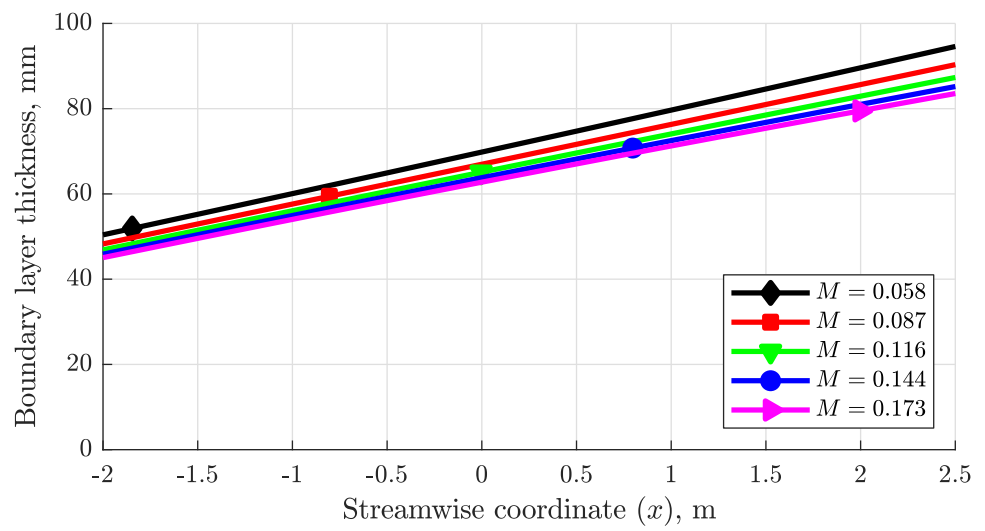

Figure 9: Boundary layer growth over the Kevlar windows at the tunnel mid-height for an empty tunnel configuration determined using the computational fluid dynamics simulations of Szőke et al. 35 .

Unlike in Amiet's model, the flow is slightly accelerated in the test section along the streamwise direction because of the increasing blockage caused by the growth of the turbulent boundary layer over the test section walls. This property of closed-section wind tunnels is usually quantified using the buoyancy parameter, which is a measure of static pressure drop per unit length along the tunnel centerline. Considering the SWT, the buoyancy was found to be approximately $15 \mathrm{~Pa} / \mathrm{m}$ at $M \approx 0.2[36$. Hence, the boundary layer in the currently evaluated empty tunnel configuration can be considered as a zero pressure gradient boundary layer. More importantly, the thickness of the boundary layer is significant due to the size of the facility used in this study. Due to the leakage flow across the pores of the Kevlar windows [2], the boundary layer thickness varies depending on the aerodynamic conditions inside the test section. While 
a detailed quantification of boundary layer properties over the flexible Kevlar walls is difficult to obtain using conventional flow measurement techniques (such as Pitot-static probe), some data is available from computational fluid dynamics (CFD) simulations of Devenport et al. [37] and Szőke et al. [35], whose results were validated against boundary layer measurements obtained immediately downstream of the Kevlar windows. An important finding of Devenport et al. [37] was that the flow over the Kevlar windows observe the behavior of a no-slip boundary layer, therefore, results obtained using a hard wall test section [36, 38, 40. could be a straightforward way to define the shear characteristics over the Kevlar windows, assuming an empty test section configuration. Using the CFD simulations of Szöke et al. [35, the boundary layer growth for an empty tunnel configuration at the tunnel mid-height is given in Fig. 9, where $x=0 \mathrm{~m}$ corresponds to the streamwise position of the LIP. To obtain the data presented in Fig. 9, the CFD simulations of Szőke et al. 35. were performed for an empty tunnel configuration incorporating the transpiration flow across the Kevlar windows. From these results, it is possible to define boundary layer (or shear layer) characteristics which are representative of the specific test cases discussed in this paper. In terms of momentum thickness Reynolds number, the CFD results show that $R e_{\theta}$ over the Kevlar windows is within the range of 20,00030,000 for $M=0.173$. Overall, this relatively high Reynolds number also confirms the significance of the boundary layer, therefore it suggests that the refraction is most likely not infinitesimal but gradual over the boundaries of the test volume, i.e. over the Kevlar windows. The combination of these effects is most likely the reason that $\varepsilon_{\tau}$ is positive and increases as the observer location departs from $\theta_{i}=90^{\circ}$ and $\psi_{i}=0^{\circ}$. Finally, the discrepancy in the arrival times remains reasonably low within $\theta_{i}=90^{\circ} \pm 30^{\circ}$, which suggests that acoustic instrumentation devices, such as beamforming microphone arrays, are best to be located within this area in the far field to minimize the flow-induced errors in the acoustic measurements. Overall, the arrival times are a relatively straightforward way to evaluate the acoustic properties of the LIP and its propagation characteristics in HAWT environments, and therefore this metric can also be used to assess some facility-specific sound propagation properties. 

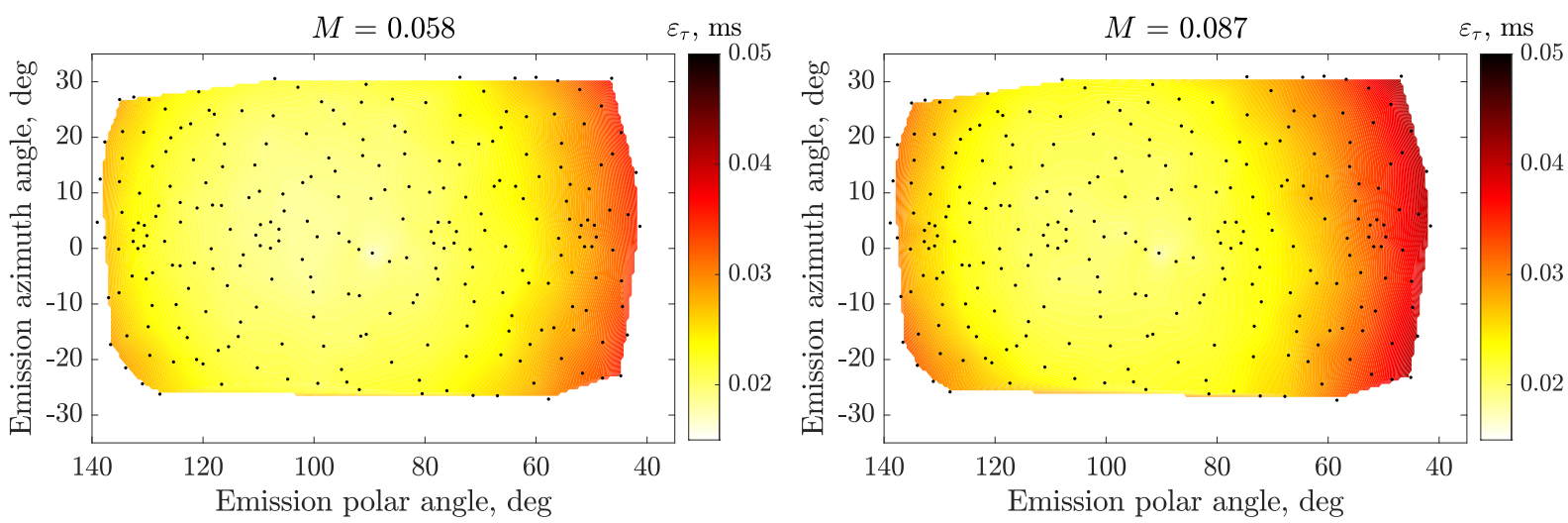

(a) Difference between measured and theoretical arrival times (b) Difference between measured and theoretical arrival times at $M=0.058$. at $M=0.087$.
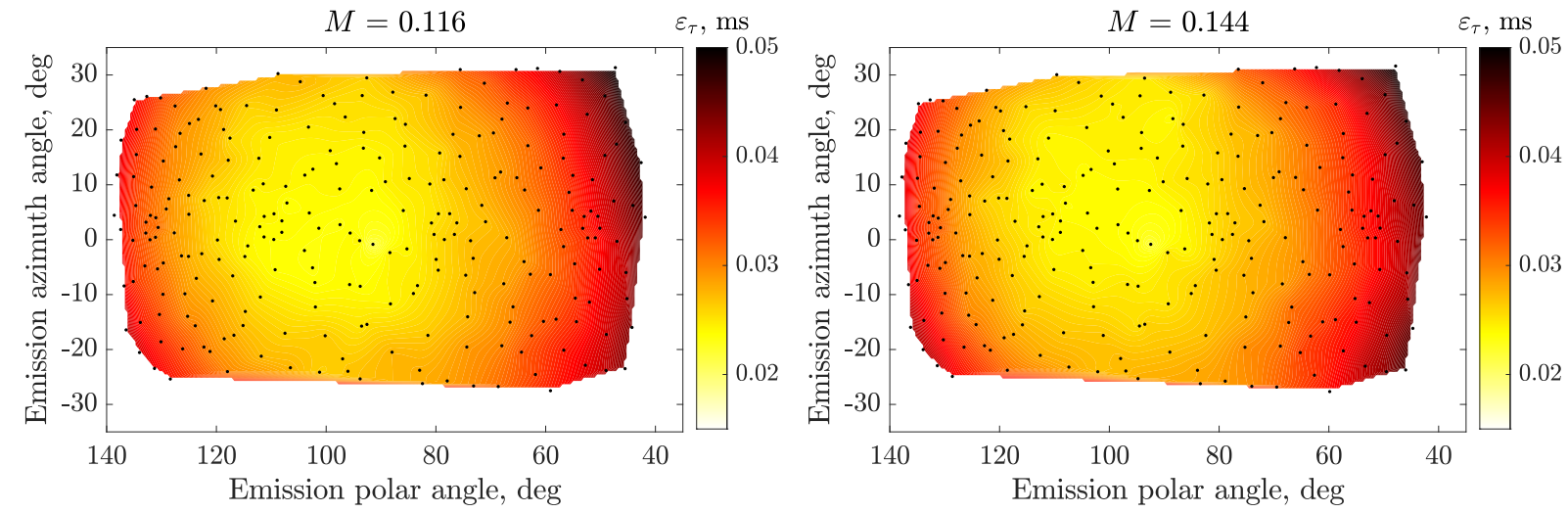

(c) Difference between measured and theoretical arrival times (d) Difference between measured and theoretical arrival times at $M=0.116$. at $M=0.144$

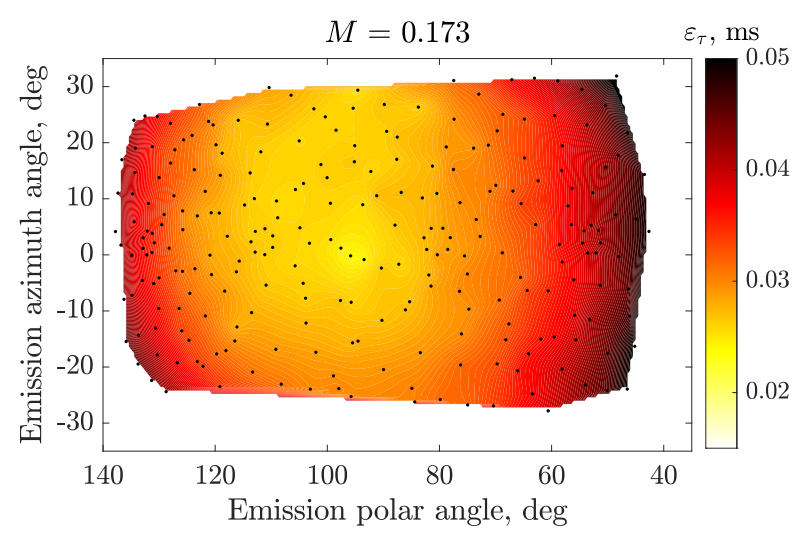

(e) Difference between measured and theoretical arrival times at $M=0.173$.

Figure 10: Arrival time discrepancy as a function of emission polar and emission azimuth angles and Mach number. The black markers indicate the locations of the microphones in the beamforming array. 


\subsection{Pressure Correction Ratio}

The error of the pressure correction ratio $\left(\varepsilon_{p}\right)$ obtained from the measurements conducted in the Stability Wind Tunnel for a range of Mach numbers is now considered. The definition of $\varepsilon_{p}$ is given in Eq. (4). In the following paragraphs, results are shown as a function of emission polar angle $\left(\theta_{i}\right)$ and emission azimuth angle $\left(\psi_{i}\right)$ and as a function of Mach number. The pressure correction ratio is also investigated as a function of frequency at 9 individual microphone locations for various Mach numbers.

Figure 11 presents the pressure correction ratio as a function of emission angles and Mach number. The pressure correction error is nearly homogeneous over the entire investigated domain and it remains very close to $0 \mathrm{~dB}$, but a moderate polar angle dependence with almost no azimuth angle dependence is observed. The error becomes slightly positive at upstream $\left(\theta_{i}>90^{\circ}\right)$ observer angles and slightly negative at downstream $\left(\theta_{i}<90^{\circ}\right)$ observer angles as the Mach number increases. Overall, the results remain within $\pm 1 \mathrm{~dB}$ which is only slightly larger than the uncertainty of the pressure levels, which ranges from \pm 0.4 to $\pm 0.8 \mathrm{~dB}$ between $M=0$ and $M=0.173$, see Figs. 6 and 7

To improve the ratio between the results and the experimental uncertainty, the pressure correction ratio has been spatially averaged along the azimuth direction $(\psi)$, with the results shown in Fig. 12 . The error $\left(\varepsilon_{p}\right)$ remains close to uncertainty bounds, namely, it stays within $\pm 0.5 \mathrm{~dB}$ between $\theta_{i}=90^{\circ} \pm 30^{\circ}$. Again, at upstream observer angles, Amiet's model underestimates the correction as $\varepsilon_{p}$ is positive, and it overestimates the correction at downstream observer angles because $\varepsilon_{p}$ is negative. A similar observation regarding $\varepsilon_{p}$ has been reported from the Quiet Flow Facility of NASA Langley [10, therefore the findings of Fig. 12 is in agreement with previous studies. This discrepancy may be caused by the differences between the experiments and model assumptions, such as, the flow speed is not constant as a function of streamwise coordinate within the test section. More importantly, the effect of the sound presumably refracting gradually within the boundary layer is neglected. In addition, while the effect of Kevlar windows on sound refraction and attenuation is assumed to cancel in the experiments, these assumptions have not yet been confirmed by previous studies and any departure from these assumptions could potentially explain the small discrepancies observed in Fig. 12 .

Finally, the the frequency-dependence of the pressure correction ratio $\left(E_{p}(f)\right)$ is considered. The current measurement approach enables the frequency-dependent investigation of the shear layer correction magnitude with the corresponding data processing approach introduced in Section 3. Amiet's shear layer method, on the other hand, does not consider the shear layer refraction in the frequency domain, therefore the modeled pressure correction is still considered as a constant in Eq. (5). The frequency-dependent pressure 

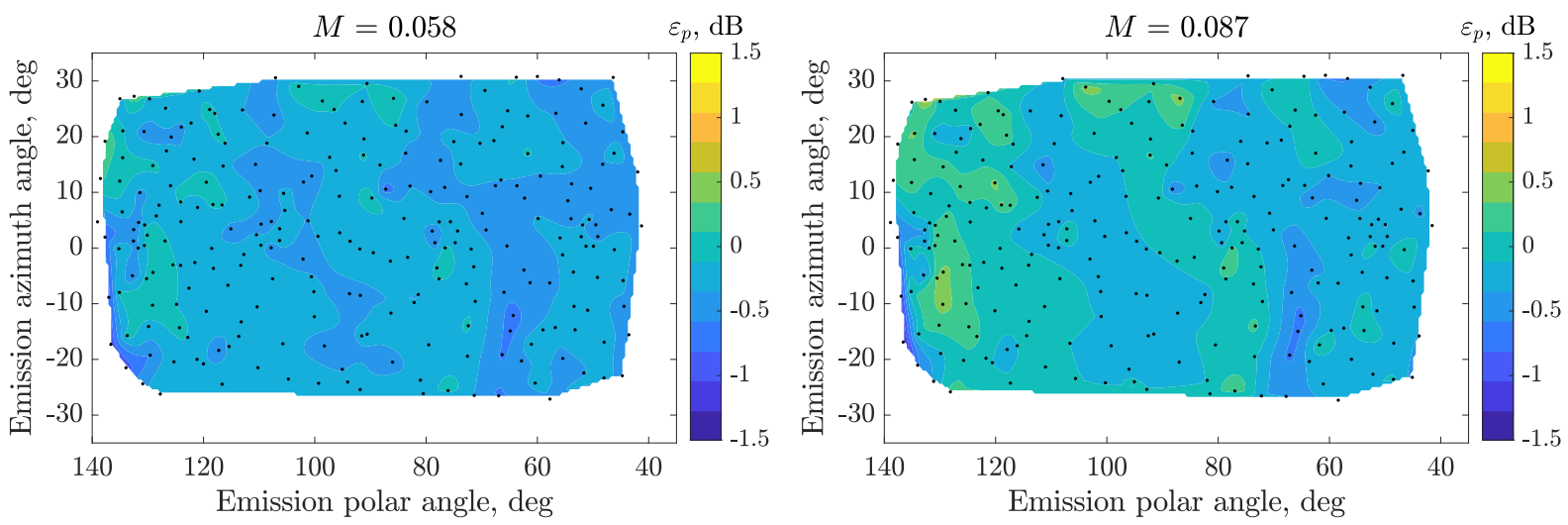

(a) Discrepancy in the pressure corrections at $M=0.058$.

(b) Discrepancy in the pressure corrections at $M=0.087$.
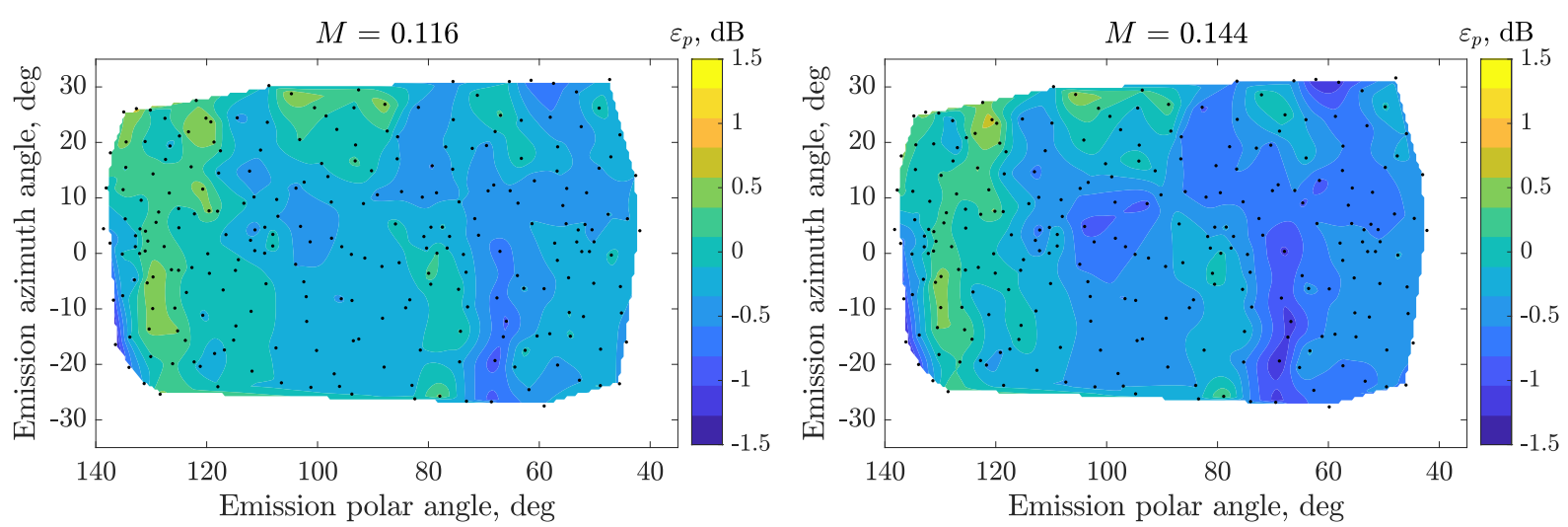

(c) Discrepancy in the pressure corrections at $M=0.116$.

(d) Discrepancy in the pressure corrections at $M=0.144$.

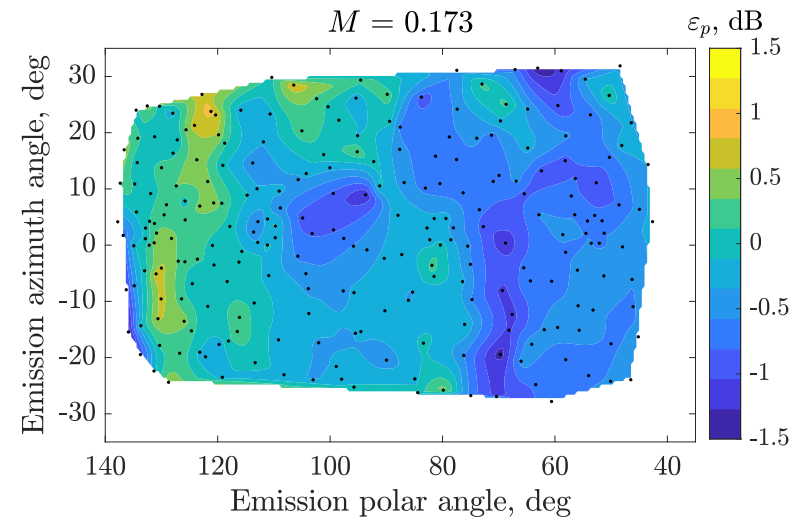

(e) Discrepancy in the pressure corrections at $M=0.173$.

Figure 11: Pressure correction discrepancy as a function of emission polar and emission azimuth angles and Mach number. The black markers indicate the locations of the microphones in the beamforming array. 


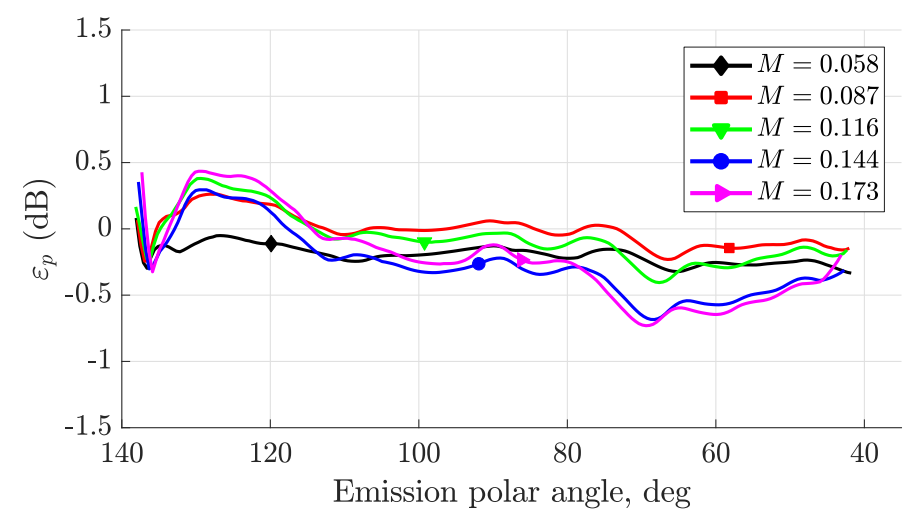

Figure 12: Pressure correction discrepancy as a function of emission polar angles and Mach number.

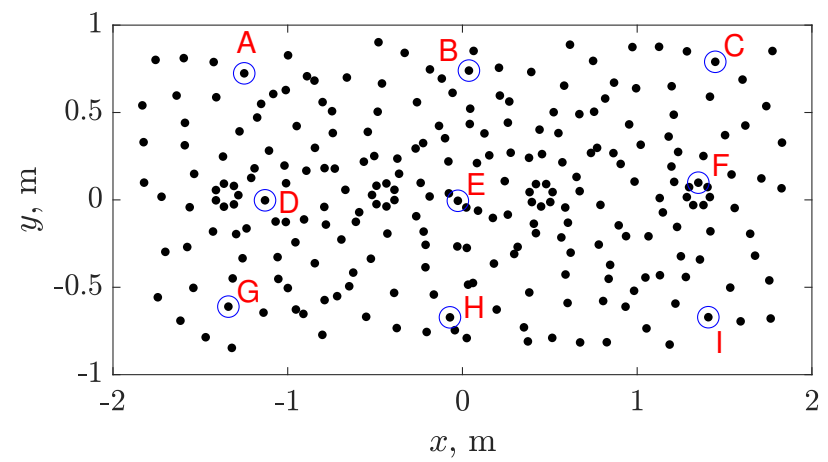

Figure 13: The far field microphone array used in this study. The circled locations indicate the microphones chosen for the frequency-dependent pressure correction analysis.

correction ratio is shown at 9 individual microphone locations (Mic. A-I), as marked in Fig. 13.

Figure. 14 presents $E_{p}$ as a function of frequency between $1 \mathrm{kHz}$ and $10 \mathrm{kHz}$. The lowest frequency is limited by the temporal length of the LIP pressure signal (0.1-0.2 ms), while at high frequencies $(f>10 \mathrm{kHz})$ the curves show a behavior dominated by the response of the microphones. The magnitude of $E_{p}(f)$ in Fig. 14 compares well to the magnitudes of $\varepsilon_{p}$ previously shown in Figs. 11 and 12 . The individual curves of pressure correction ratios in Fig. 14 remain well within one decibel over the entire domain of frequencies investigated here, regardless of the Mach number. The most important observation regarding $E_{p}(f)$, therefore, is that the frequency dependence of the sound ray does not change between $1 \mathrm{kHz}$ and $10 \mathrm{kHz}$ as it propagates from the LIP to the far field microphones, i.e. the interaction between the boundary layer turbulence and the sound field and the refraction itself does not modify the frequency content of the sound field between $1 \mathrm{kHz}$ and $10 \mathrm{kHz}$. 

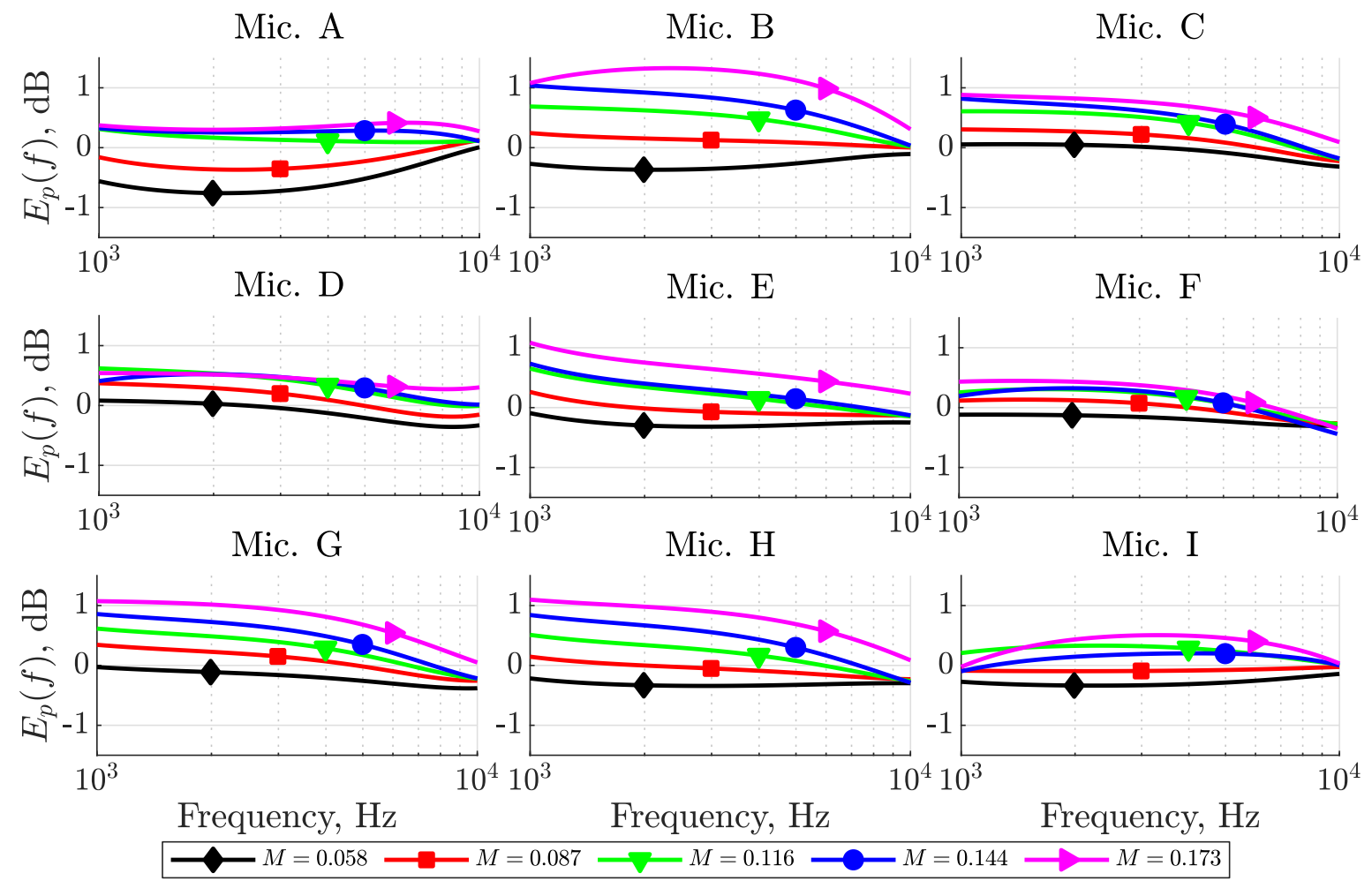

Figure 14: Pressure correction discrepancy as a function of frequency and Mach number at 9 individual far field microphone locations. 


\section{Conclusions}

This paper presents an aeroacoustic investigation of an impulse point source generated using laser-induced plasma (LIP). The LIP was positioned to the center of the test section at Virginia Tech's Stability Wind Tunnel, which is a Kevlar-walled hybrid anechoic wind tunnel (HAWT). The sound of the LIP was captured using a 251-element far field microphone array while the flow speed in the test section was varied. The sound emission time instant of the LIP was identified using a photodetector. The formulation of the sound wave in the vicinity of the LIP was captured using high-speed Schlieren imaging at ambient, no flow conditions.

The experimental approach enabled the comparison of sound propagation characteristics to Amiet's model of shear-layer refraction [8]. Namely, the arrival time of the pressure waves (source to microphones) and the pressure correction magnitude were assessed, both experimentally and analytically. The high-speed Schlieren imaging revealed that the sound of the LIP reaches the far field microphones over a shorter time duration because the sound wave around the LIP travels at a supersonic wave speed for a short period of time. The discrepancy was found to be comparable to the temporal resolution of the aeroacoustic experiments.

An uncertainty analysis on the metrics of shear-layer refraction was considered. The uncertainty of the arrival times was limited by the temporal resolution of the microphone signals and this uncertainty was not significantly affected by the flow speed. The uncertainty of the pressure levels was found to be limited by the uncertainty of the microphones while it increased at shallow observer angles at higher flow speeds due to the interaction between the boundary layer flow and the sound rays.

The discrepancy between the measured and theoretical arrival times were found to be primarily dependent on the polar angles and only a moderate azimuth angle dependence was found. The discrepancy increased with flow speed and it was always positive, therefore, Amiet's model was found to underestimate the arrival time of the pressure waves despite the fact that the supersonic wave propagation was expected to cancel by definition. The discrepancy between the measured and theoretical pressure correction was found to have a uniform distribution across the azimuth observer angles. The discrepancy became positive at upstream polar observer angles and negative at downstream polar observer angles with increasing flow speed. Finally, the frequency-dependent analysis of the pressure correction discrepancy revealed that the refraction does not affect the frequency content of the sound between $1 \mathrm{kHz}$ and $10 \mathrm{kHz}$.

\section{Declaration of interest}

The authors report no conflict of interest. 


\section{Acknowledgments}

The authors would like to thank Christopher J. Bahr for the useful discussions during this research study. The constructive comments from Forence Hutcheson are also appreciated. The authors would like to acknowledge K. Todd Lowe and Kyle Daniel for their support in designing the laser-optical components and performing the high-speed Schlieren experiments. The technical support provided by AVEC, Inc., in particular by Patricio Ravetta, is much appreciated. The financial support from the Stability Wind Tunnel is greatly appreciated.

\section{References}

[1] W. M. Humphreys Jr., T. F. Brooks, Noise spectra and directivity for a scale-model landing gear, International Journal of Aeroacoustics 8 (5) (2009) 409-443.

[2] W. J. Devenport, R. A. Burdisso, A. Borgoltz, P. A. Ravetta, M. F. Barone, K. A. Brown, M. A. Morton, The Kevlar-walled anechoic wind tunnel, Journal of Sound and Vibration 332 (17) (2013) 3971-3991.

[3] J. Lv, N. Wang, D. Liao, Y. Yu, X. Gao, Study on acoustically transparent test section of aeroacoustic wind tunnel, Journal of Applied Mathematics and Physics 6 (01) (2018) 1-10.

[4] L. Li, P. Liu, H. Guo, Y. Hou, X. Geng, J. Wang, Aeroacoustic measurement of 30P30N high-lift configuration in the test section with Kevlar cloth and perforated plate, Aerospace Science and Technology 70 (2017) 590-599.

[5] M. Debrouwere, An assessment of acoustically transparent wind tunnel walls, in: Delft University of Technology, MSc Thesis, 2013.

[6] K. Brown, W. Devenport, A. Borgoltz, Aircraft noise generation and assessment: exploitation of hybrid anechoic wind tunnels for aeroacoustic and aerodynamic measurements, CEAS Aeronautical Journal 10 (1) (2019) $251-266$.

[7] H. Ura, M. Shigemi, T. Hirotani, T. Homma, Wall interference correction method for Kevlar wall test section, Journal of Aircraft 57 (5) (2020) 889-900

[8] R. K. Amiet, Refraction of sound by a shear layer, Journal of Sound and Vibration 58 (1978) $467-482$.

[9] C. Bahr, N. S. Zawodny, T. Yardibi, F. Liu, D. Wetzel, B. Bertolucci, L. Cattafesta, Shear layer time-delay correction using a non-intrusive acoustic point source, International Journal of Aeroacoustics 10 (5-6) (2011) 497-530.

[10] M. Szőke, C. J. Bahr, F. V. Hutcheson, W. J. Devenport, Characterization of hybrid wind tunnel environments using laser-induced acoustic sources, in: AIAA Scitech 2020 Forum, AIAA-2020-1253.

[11] C. J. Bahr, F. V. Hutcheson, D. J. Stead, Assessment of unsteady propagation characteristics and corrections in aeroacoustic wind tunnels using an acoustic pulse, in: 2018 AIAA/CEAS Aeroacoustics Conference, AIAA-2018-3118

[12] J. Zhang, X. Wang, J. Zhang, C. J. Doolan, J. R. Fischer, D. Moreau, A study of shear-layer corrections and a tensioned fabric wall for the localization of sound sources in wind tunnel, in: 25th AIAA/CEAS Aeroacoustics Conference, AIAA2019-2717.

[13] A. W. Miziolek, V. Palleschi, I. Schechter, Laser induced breakdown spectroscopy, Cambridge University Press, 2006.

[14] T. G. Jones, M. K. Hornstein, A. C. Ting, Z. W. Wilkes, Intense underwater laser acoustic source for navy applications., The Journal of the Acoustical Society of America 125 (4) (2009) 2556-2556. 
[15] N. Hosoya, M. Nagata, I. Kajiwara, Acoustic testing in a very small space based on a point sound source generated by laser-induced breakdown: Stabilization of plasma formation, Journal of Sound and Vibration 332 (19) (2013) 4572-4583.

[16] A. K. Soh, Y. Sun, D. Fang, Vibration of microscale beam induced by laser pulse, Journal of sound and vibration 311 (1-2) (2008) 243-253.

[17] F. Huda, I. Kajiwara, N. Hosoya, Damage detection in membrane structures using non-contact laser excitation and wavelet transformation, Journal of Sound and Vibration 333 (16) (2014) 3609-3624.

[18] K.-S. Rossignol, J. Delfs, Analysis of the noise shielding characteristics of a NACA0012 2D wing, in: 22nd AIAA/CEAS Aeroacoustics Conference, AIAA-2016-2795.

[19] K.-S. Rossignol, M. Pott-Pollenske, J. Delfs, J. Silbermann, J. M. Pereira Gomes, Investigating noise shielding by unconventional aircraft configurations, in: 23rd AIAA/CEAS Aeroacoustics Conference, AIAA-2017-3195.

[20] D. Ernst, C. Spehr, T. Berkefeld, Decorrelation of acoustic wave propagation through the shear layer in open jet wind tunnel, in: 21st AIAA/CEAS Aeroacoustics Conference, AIAA-2015-2976.

[21] K. Pascioni, A. Colangelo, L. Cattafesta, Acoustic corrections for a Kevlar wall wind tunnel using a pulsed-laser point source, in: 27th International Congress on Sound and Vibration, London, UK, 2017.

[22] K.-S. Rossignol, J. Delfs, F. Boden, On the relevance of convection effects for a laser-generated sound source, in: 21st AIAA/CEAS Aeroacoustics Conference, AIAA-2015-3146.

[23] K.-S. Rossignol, M. Lummer, J. Delfs, Validation of DLR's sound shielding prediction tool using a novel sound source, in: 15th AIAA/CEAS Aeroacoustics Conference (30th AIAA Aeroacoustics Conference), AIAA-2009-3329.

[24] Q. Qin, K. Attenborough, Characteristics and application of laser-generated acoustic shock waves in air, Applied Acoustics 65 (4) (2004) 325-340.

[25] G. Ostrovskaya, et al., Laser spark in gases, Soviet Physics Uspekhi 16 (6) (1974) 834.

[26] C. Leela, S. Bagchi, V. R. Kumar, S. P. Tewari, P. P. Kiran, Dynamics of laser induced micro-shock waves and hot core plasma in quiescent air, Laser and Particle Beams 31 (2) (2013) 263-272.

[27] M. S. Bak, L. Wermer, S.-k. Im, Schlieren imaging investigation of successive laser-induced breakdowns in atmosphericpressure air, Journal of Physics D: Applied Physics 48 (48) (2015) 485203.

[28] M. Thiyagarajan, J. Scharer, Experimental investigation of ultraviolet laser induced plasma density and temperature evolution in air, Journal of applied physics 104 (1) (2008) 013303.

[29] G. I. Taylor, The formation of a blast wave by a very intense explosion I. Theoretical discussion, Proceedings of the Royal Society of London. Series A. Mathematical and Physical Sciences 201 (1065) (1950) 159-174.

[30] J. Von Neumann, The point source solution, John Von Neumann Collected Works, Vol.6 (1963) $219-237$.

[31] C. J. Bahr, N. S. Zawodny, B. Bertolucci, J. Li, M. Sheplak, L. N. Cattafesta, A plasma-based non-intrusive point source for acoustic beamforming applications, Journal of Sound and Vibration 344 (2015) 59-80.

[32] Y. D. Mayer, H. K. Jawahar, M. Szőke, S. A. S. Ali, M. Azarpeyvand, Design and performance of an aeroacoustic wind tunnel facility at the university of Bristol, Applied Acoustics 155 (2019) 358-370.

[33] Mathworks, Inc., Matlab user's guide URL https: //www . mathworks . com/help/matlab/

[34] S. Glegg, W. Devenport, Aeroacoustics of low Mach number flows: fundamentals, analysis, and measurement, Academic Press, 2017.

[35] M. Szőke, W. Devenport, A. Borgoltz, C. Roy, T. Lowe, Advanced boundary simulations of an aeroacoustic and aerody- 
namic wind tunnel, in: Advanced Wind Tunnel Boundary Simulation II. STO-MP-AVT-388, 2021.

[36] M. Szőke, V. Vishwanathan, T. Loeschen, A. Gargiulo, D. J. Fritsch, J. E. Duetsch-Patel, A. Borgoltz, C. J. Roy, K. T. Lowe, W. J. Devenport, Developing a numerical model of the virginia tech stability wind tunnel for uncertainty quantification based on real-world geometry, in: AIAA Scitech 2020 Forum, 2020, pp. AIAA-2020-0343.

[37] W. Devenport, K. Brown, A. Borgoltz, E. Paterson, C. Bak, N. Sørensen, A. Olsen, M. Gaunaa, A. Fischer, C. Grinderslev, Advanced wind tunnel boundary simulation for kevlar wall aeroacoustic wind tunnels, in: Advanced Wind Tunnel Boundary Simulation STO-MP-AVT-284, 2018.

[38] L. A. Joseph, N. J. Molinaro, W. J. Devenport, T. W. Meyers, Characteristics of the pressure fluctuations generated in turbulent boundary layers over rough surfaces, Journal of Fluid Mechanics 883 (2020).

[39] T. Meyers, J. B. Forest, W. J. Devenport, The wall-pressure spectrum of high-reynolds-number turbulent boundary-layer flows over rough surfaces, Journal of Fluid Mechanics 768 (2015) 261-293.

[40] V. Vishwanathan, M. Szőke, J. E. Duetsch-Patel, A. Gargiulo, D. J. Fritsch, A. Borgoltz, C. J. Roy, K. T. Lowe, W. J. Devenport, Aerodynamic design and validation of a contraction profile for flow field improvement and uncertainty quantification in a subsonic wind tunnel, in: AIAA SciTech 2020 Forum, 2020, pp. AIAA-2020-2211. 